Internist $2021 \cdot 62: 1215-1219$

https://doi.org/10.1007/s00108-021-01205-8

Angenommen: 14. Oktober 2021

Online publiziert: 22. Oktober 2021

(c) Der/die Autor(en) 2021

\section{Gendern in der Medizin - ein Appell}

\author{
Markus M. Lerch \\ LMU Klinikum, München, Deutschland
}

Die Deutsche Gesellschaft für Innere Medizin (DGIM) wurde vor 140 Jahren in Wiesbaden gegründet. Sie erfreut sich trotz ihres Alters einer exzellenten Gesundheit und kann mit mehr als 28.000 Mitgliedern gegenüber den Wechselfällen der Geschichte als durchaus robust und beständig angesehen werden. Die DGIM legte schon immer größten Wert auf Diversität. Unter ihren Mitgliedern, Ehrenmitgliedern und Vorsitzenden sind 27 Nationen, 29 Berufe und Fachrichtungen und 10 Nobelpreisträger vertreten. $46 \%$ der Mitglieder sind Frauen, unter den 1500 studentischen Mitgliedern sind es $57 \%$ und unter den Ärzten in der Weiterbildung $60 \%$. Liest man die stolze Geschichte der DGIM, fällt auf, dass sich unter den 127 Vorsitzenden genau zwei Frauen und unter den 223 Ehrenmitgliedern zwei weitere Frauen befinden. Möglicherweise haben es die Gremien der DGIM geschafft, Frauen für herausgehobene Positionen als ungeeignet zu betrachten, oder sie haben ihnen einfach ausgeredet, sich um solche Ämter zu bewerben. Andererseits könnten sich die Frauen in unseren Reihen beim Aufruf für die Vergabe von Orden und Ämtern nicht angesprochen gefühlt haben. Gegen Letzteres gibt es heute ein Mittel. Das wäre das Gendern.

Für alle, die die seit 40 Jahren entwickelte feministische Sprachkritik nicht mitbekommen haben sollten: die Verwendung des männlichen Geschlechts bei der Benennung und Ansprache von Personen führt dazu, dass der Hörer beim Begriff „Flugbegleiter" immer das Bild eines Mannes vor Augen hat und Frauen sich nicht wirklich angesprochen, bestenfalls "mitgemeint" fühlen und sich deshalb praktisch nie um den ehrenamtlichen Vorsitz der Weiterbildungskommission der DGIM beworben haben. Gendern oder eine konse- quent geschlechtsneutrale Sprache schafft da Abhilfe.

\section{Die Werkzeuge des Genderns}

Gendern bedeutet, dass man im gesprochenen oder geschriebenen Satz entweder alle männlichen Bezeichnungen durch (nur im Plural) geschlechtsneutrale Partizipien ersetzt oder Begriffe wählt, die Männer und Frauen gleichzeitig ansprechen (so man in binären Geschlechterzuschreibungen denkt). Aus Studenten werden Studierende und aus Mitarbeitern werden Mitarbeitende. Wer lange genug an einer Hochschule unterwegs war, bedauert ein wenig den Verlust an sprachlicher Präzision, weil ihm Mitarbeiter einfallen, die nicht unbedingt als Mitarbeitende in Erscheinung getreten sind, schon gar nicht, wenn sie streiken. „Streikende Mitarbeitende" sind im Deutschen ein offensichtlicher Widerspruch, "streikende Mitarbeiter " hingegen nicht. Längst nicht alle Studenten sind Studierende. Dies ist aber in der Medizin eine Ausnahmeerscheinung und soll in den Geisteswissenschaften verbreiteter sein. Eine andere Lösung ist, dass man bei der Nennung immer beide Geschlechter ausschreibt: Internistinnen und Internisten, Ärztinnen und Ärzte. Das funktioniert, verlängert aber manche Texte deutlich. In der DGIM immer dann, wenn wir aus Gründen der Inklusion sehr häufig Mitglieder aus allen 11 Teilfachbereichen direkt ansprechen (Nephrologinnen und Nephrologen, Endokrinologinnen und Endokrinologen etc.). Zusätzliche inhaltliche Informationen übermittelt das nicht. Oder man nutzt neue Schreibweisen wie Internist/nnen, Internist_innen (Gender-Gap), Internist/innen oder Internist:innen. 


\section{Gendern an Universitäten}

Im akademischen Umfeld erfreut sich das Gendern großer Beliebtheit und breitet sich rasant aus [1]. Es liegt voll im Zeitgeist, signalisiert, dass man Gleichstellung ernst nimmt, wirkt modern und aufgeschlossen, belebt den akademischen Alltag und zahlreiche Diskussionsforen und zeigt, dass man unter gar keinen Umständen patriarchalischen Strukturen Vorschub leistet auch nicht in der Sprache [2]. Wer gendert, setzt sich nie dem Verdacht aus, auf der ideologisch falschen Seite zu stehen. An einigen Universitätsstandorten werden unterschiedliche Vorgaben zum Gendern je nach Fakultät gemacht, was regelmäßig eine Prüfung nach sich zieht, ob es sich um ein Dokument für die Universität oder die medizinische Fakultät handelt, weil sonst die jeweilige Verordnung zum Gendern verletzt wurde. Promotionsarbeiten von Promovierenden zu so rätselhaften Themen wie „Betalaktamaseaktivität in Hepatozyten" werden dort konsequent abgelehnt, wenn sie nicht durchgängig gegendert wurden. Der Hepatozyt ist männlich. Hepatozyten von Frauen dürfen nicht nur mitgemeint sein. Die Universität Leipzig geht einen Schritt weiter und hat sich entschieden, statt Frauen sichtbar Männer unsichtbar zu machen, und es dürfen grundsätzlich nur noch weibliche Formen, im Sinne eines generischen Femininums, angewendet werden. Herr Dr. Müller gehört jetzt zur Statusgruppe der Professorinnen. Eine sprachliche Weiterentwicklung ist die Schreibweise als Internist*innen, wobei dem Gendersternchen zugeschrieben wird, eben nicht nur Männer und Frauen, sondern auch nicht binäre oder fluide Geschlechterrollen zu bezeichnen und damit anzusprechen.

Liebe Leser*innen, die DGIM könnte das auch tun. Es könnte sich als viel praktikabler erweisen zu gendern, als dafür Sorge zu tragen, dass auch mal Frauen zu Präsident*innen gewählt werden oder dass wichtige Kommissionen der DGIM paritätisch mit Männern und Frauen besetzt werden. Nur weil der Wissenschaftsrat und die Deutsche Forschungsgemeinschaft das tun und dadurch eine große Nachhaltigkeit in der Gleichstellung erreicht haben, muss man sich so was nicht unbedingt zumuten. Die DGIM könnte alle
Chef*ininternist*innen in Zukunft gegendert ansprechen, damit jedermann sich in ihrer Wahrnehmung berücksichtigt fühlt.

\section{I) Gendern ist bewusst darauf ausgelegt, den/die Leser*in oder den/die Zuhörer*in zu irritieren}

Gendern ist famos. Es ist bewusst darauf ausgelegt, den/die Leser*in oder den/die Zuhörer*in zu irritieren [1]. Es erinnert uns auch in Texten zur Nachtdienstbelastung im Krankenhaus oder zum Burn-out in der Niederlassung konsequent an weiterbestehende gesellschaftliche Benachteiligungen von Frauen. Selbst wenn diese von den adressierten Missständen in gleicher Weise betroffen sind wie ihre männlichen Kollegen. Die gesamte Ärzt*innenschaft könnte somit davon profitieren. Endlich würde die DGIM, einschließlich ihres Vorstands aus ausschließlich (mittel)alten weißen Männern, mit der Gleichstellung Ernst machen. Ein Anfang ist bereits getan. Die „Jungen Internisten“ wurden jetzt in „Junge DGIM“ umbenannt (das war einfach). Auch der Berufsverband der Internisten könnte eventuell mit der bereits erfolgten Umbenennung in BDInternist*innen bei seiner Mitgliederversammlung durchkommen.

\section{Kritik am Gendern}

Man müsste aber fest mit Kritik aus den Reihen der DGIM-Mitglieder rechnen. In den neuen Bundesländern, wo sich Frauen viel früher als im Westen Zugang zu den technischen Berufen erkämpft haben, empfinden viele die Bezeichnung als Arzt, Ingenieur oder Traktorist als angemessen und die weibliche Form als herabsetzend. In angelsächsischen Ländern wollen Frauen nicht mehr als "actress" oder "lawyeress", sondern mit der männlichen Bezeichnung "actor" oder "lawyer" bezeichnet werden, weil sie sonst nur einer Unterkategorie angehören. Für Italien gilt das Gleiche und im Schwedischen gelten die "movierten“ weiblichen Formen von generisch männlichen Berufsbezeichnungen als veraltet. In Deutschland reiht sich Dr. Angela Merkel für die meisten Menschen in die Liste der Bundeskanzler (und nicht der Bundeskanzler*innen oder Bundeskanzlerinnen) ein. Viele Mitglieder empfinden Gen- dern als Bevormundung, als sprachpädagogisches "Social Engineering", als übergriffig und bevormundend, als Legitimation für identitären Aktivismus unter dem Deckmantel des positiv besetzten Ziels der Gleichstellung. Sie weisen dreist daraufhin, dass es für das Gendern keinerlei gesetzliche Grundlage gibt und ein "durchgegendertes" Gesetz des Justizministeriums (SPD, Ministerin Frau) vom Innenministerium (CSU, Minister Mann) gestoppt wurde, weil es sonst keine universelle Wirkung für alle Menschen entfaltet hätte. Man könnte unterstellen, dass es sich bei all diesen Kritikern um alte weiße Männer handelt, denen es ausschließlich um die Erhaltung patriarchalischer (Sprach-)Strukturen geht [2].

Dabei liegt man aber falsch. Repräsentative Umfragen haben ergeben, dass die Mehrheit der Bundesbürger, und auch die Mehrheit der Bundesbürgerinnen, das Gendern ablehnt [3]. Ähnliches gilt auch für eine nichtrepräsentative Umfrage unter den weiblichen Mitarbeiterinnen der DGIM-Geschäftsstelle, die mehrheitlich nicht gendern wollen.

Versuche, Sprache politisch zu instrumentalisieren, hat es schon immer gegeben [4]. Heute handelt es sich dabei meist um den Austausch von herabsetzenden Bezeichnungen für Minderheiten. Dies wird aber als viel weniger eingreifend empfunden als das konsequente Gendern. Die deutsche Sprache wird zudem von allein immer genderbewusster und geschlechtergerechter. Begriffe wie Feuerwehrleute, Fachkraft oder die unhandlichen Auszubildenden (zumindest als Azubis) sind längst umgangssprachlich. Bei der direkten Ansprache oder auf Formularen werden Männer und Frauen fast immer spezifisch (statt generisch) angesprochen. Das ist überall akzeptiert und wird natürlich auch von der DGIM längst praktiziert. Ob sich dagegen das konsequente Gendern durchsetzen oder jemals allgemeine Akzeptanz finden wird, bleibt abzuwarten - in einer großen bayerischen Volkspartei eher nicht [5].

\section{Kampf um das generische Maskulinum}

Der heilige Gral sowohl der Sprachfeministen als auch der vehementen Bekämpfer 
Hier steht eine Anzeige.

黑 Springer 
des Genderns ist das generische, inklusive Maskulinum. Wann immer sich die DGIM an säumige Beitragszahler wendet und damit alle Mitglieder - Männer, Frauen und Diverse - meint und die geforderte Überweisung erwartet, verwendet sie das generische Maskulinum. Ziel des Kampfs ist, dieses für immer aus der Sprachkultur zu verbannen oder als einzig korrekte Anrede von Personengruppen beiderlei Geschlechts zu erhalten.

Nutzern des generischen, inklusiven Maskulinums wird unterstellt, sie bewegten sich außerhalb der "Political Correctness" oder seien patriarchalische Gleichstellungsgegner jenseits des akzeptierten ideologischen Spektrums. Verwender*innen des Gendersternchens wird dagegen vorgeworfen, sie betrachteten jedes dieser Sternchen als Landgewinn im sprachfeministischen Grabenkampf oder bei der Durchsetzung des betreuten Schreibens und Sprechens. Sie verstünden nicht den Unterschied zwischen Sexus (biologisches Geschlecht) und Genus (grammatikalisches Geschlecht): der Kuchen, die Sonne, der Mond und die Stadt hätten ja wohl kaum etwas mit der Ausdifferenzierung von Genitalien zu tun. Sie zu gendern verstümmele über Jahrhunderte gewachsene Sprachstrukturen.

\section{Blick in die Geschichte}

Das Problem ist, so alt ist das generische Maskulinum möglicherweise gar nicht. Ein Blick in die Sprachlehren vergangener Tage lohnt sich [6]. In Texten und Rechtstexten des Mittelalters kommen Frauen nicht besonders häufig vor. Wenigstens mal als Straftäter, wie in H. Kramers Hexenhammer, aber der ist leider noch auf Lateinisch abgefasst. Lange galten Frauen als Teil des Hausstands ihrer Ehemänner oder Väter und damit als Besitz. Besitz braucht keine eigenen Rechte oder Rechtsvorschriften. In der Renaissance wird das anders und die Rechtsstellung der Frauen bessert sich. Da ihre Rechte sich aber immer von denen der Männer unterscheiden, werden sie auch immer spezifisch (und nie generisch) erwähnt. In den Grammatiken dieser Zeit finden sich aber schon Einteilungen wie das "genus communis" und das "genus promiscuus" und Beispiele wie "der gelerte man, die gelerte fraw, das geler- te Mensch" [7]. Im Barock wird es bunter und für das grammatische Geschlecht wird vorgeschlagen: männlich, weiblich, unbenannt, zweierley und allerley [8]. Besser geht geschlechtergerechtes Sprachverständnis kaum und auch die LGBTQ*-Community könnte sich dieser Einteilung wahrscheinlich vorbehaltlos anschließen. Als „Entdecker" des generischen Maskulinums gilt der geniale Sprachforscher (und Märchensammler) Jacob Grimm in der Mitte des 19. Jahrhunderts, der zudem das Maskulinum für das „lebendigste, kräftigste und ursprünglichste" der grammatikalischen Geschlechter hält [9]. Natürlich sagt Schillers Maria Stuart „ich bin euer König“, weil Königin ja auch „nur" die Frau des Königs ohne eigenen Herrschaftsanspruch meinen könnte. Erst mit zunehmender Gleichstellung in den Verfassungen des Norddeutschen Bundes, der Weimarer Republik und dann vollständig in denen der Bundesrepublik, der DDR und Österreichs verschwinden die Frauen zunehmend. Dann komplett in den Dudenausgaben der 60er-Jahre des 20. Jahrhunderts. Weil ja alle Menschen gleich sind, sind auch alle mit dem generischen Maskulinum gemeint. Für Frauen bedeutete dies gleichzeitig einen Zuwachs an Geltung und Prestige. Man hatte die Männerdomänen erobert und nannte männliche Berufsbezeichnungen stolz sein eigen (Actor, Arzt, Traktorist). Während die Anrede Frau Geheimrat oder Frau Doktor sich vor hundert Jahren immer auf die Frau vom Geheimrat oder die Frau vom Doktor bezog (für die Rettung des Genitivs kämpfen wir an anderer Stelle), wurde man jetzt selbst Doktor (seltener Geheimrat).

\section{Sichtbarkeit statt Prestige}

Mit Arzt-Prestige ist es aber nicht mehr weit her, wenn 70-80\% eines Medizinstudiengangs heute Ärztinnen werden. Und plötzlich spielt Sichtbarkeit, zumindest für einen Teil der Frauen, eine viel größere Rolle. Prestige für männliche Berufsbezeichnungen kommt aus der Mode, wenn Frauen diese nicht nur gleichberechtigt ausüben, sondern sogar dominieren.

Anders als in Frankreich, mit der Verbindlichkeit seiner Académie française, oder den gesetzlichen Sprachvorschriften in England seit 1850 lassen sich die Spre- cher des Deutschen kaum vorschreiben, wie sie zu sprechen oder zu schreiben haben. Die Widerstände gegen die Rechtschreibreformen von 1996 und 2006 sind eindrucksvolle Beispiele.

Wer sich also heute als Präsident einer Fachgesellschaft mit geschlechtergerechter Sprache auseinandersetzt, kann nur alles falsch machen.

\section{DGIM so divers wie nie}

Im Vorstand der DGIM gibt es inzwischen starke Befürworter und entschiedene Gegner des Genderns (und sogar Zwischenstufen). Vielleicht lässt sich das dadurch erklären, dass kaum eine Fachgesellschaft so jung, dynamisch, weiblich und mitgliederstark ist wie die DGIM. Trotz des Stolzes auf eine Tradition alter weißer Männer ist die DGIM offenbar so divers, dass es auch mal männliche Kulturfeministen in den Vorstand spült. Es wäre möglich, sogar wahrscheinlich, dass eine Entscheidung für das Komplettgendern weder vom Vorstand noch von unseren Mitarbeiter:innen noch von unseren Mitgliedern akzeptiert würde. Eine vollständige Genderverweigerung und die konsequente Rückkehr zum generischen, inklusiven und neutralisierenden Maskulinum würden aber von vielen Kolleg*innen als Affront empfunden und ihnen Sichtbarkeit vorenthalten.

\section{Aufruf zu sprachlicher Toleranz}

Als DGIM-Vorsitzender darf man sich (zumindest ein Jahr lang) gewisse Freiheiten herausnehmen. Deshalb erlaube ich mir folgenden Vorschlag: Die Mitteilungen der DGIM aus Vorstand, Ausschuss und den Kommissionen stammen üblicherweise aus der Feder ganz verschiedener Autorinnen und Autoren und werden meist in einen einheitlichen Stil redigiert. Damit könnte man einfach aufhören. Solange Texte klar und verständlich sind, auch für Nichtakademiker*innen, für Kolleg:innen, deren Muttersprache nicht Deutsch ist, und auch für alte weiße Männer, könnte man sich einfach mal sprachliche Toleranz erlauben und experimentieren. Wenn die DGIM in ihren Texten gendert, könnte sie dies bewusst tun, um Inklusion und Diversität besonders zu kennzeichnen, aber vielleicht nicht konsequent, 
konsistent oder durch Vorgaben der Fachgesellschaft erzwungen. Man könnte sich in gleicher Weise in Toleranz gegenüber den Nutzern des generischen Maskulinums und Nutzer*innen des Gendersternchens üben. Wer Gendern vorschreibt [10] oder Gendersternchen sanktioniert [11], kann sich bisher weder auf eine demokratische Legitimierung noch auf eine gesetzliche Regelung berufen. Würden wir auf diese Weise Erfahrung mit verschiedenen Formen geschlechtergerechter Sprache sammeln, wären die Diskussionen der Zukunft vielleicht weniger ideologisch aufgeladen und durch praktische Erfahrung geprägt.

Bis zu dem Zeitpunkt, an dem der DGIMVorstand dann eine abschließende Bewertung und Festlegung vornimmt, könnten wir uns ja mal mit Gleichstellung in unseren Gremien beschäftigen.

Liebe Kolleginnen und Kollegen, ich freue mich auf Ihr Feedback (info@ dgim.de). Bis dahin versuche ich, verständlich zu bleiben, und hoffe, Sie verstehen mich.

Markus M. Lerch, München

Vorsitzender der DGIM

\section{Korrespondenzadresse}

\section{Markus M. Lerch}

LMU Klinikum

München, Deutschland

Markus.Lerch@med.uni-muenchen.de

Interessenkonflikt. M.M. Lerch gibt an, dass kein Interessenkonflikt besteht.

\section{Literatur}

1. Payr F (2021) Von Menschen und Mensch*innen. Springer, Berlin, Heidelberg, New York

2. Stefanowitsch A (2018) Eine Frage der Moral. Warum wir politisch korrekte Sprache brauchen. Dudenverlag, Berlin

3. Schmoll H (2019) Ungeliebter Stern. Mehrheit der Deutschen lehnt gendergerechte Sprache ab (FAZ 1.4.2019)

4. Klemperer V (2020) LTI - Notizbuch eines Philologen. Reclam, Ditzingen

5. Glas A (2021) Söder und der Genderstern. Süddeutsche Zeitung online. https://www.sueddeutsche. de/bayern/soeder-universitaeten-gendernsprache- genderstern-1.5412059? reduced=true. Zugegriffen: 16. Sept. 2021.

6. Doleschal U Das generische Maskulinum im Deutschen. Linguistik online, Wien. https://doi. org/10.13092/lo.11.915. https://bop.unibe.ch/ linguistik-online/article/view/915. Zugegriffen: 16. Sept. 2021.
7. Oelinger Albertus. Underricht der Hoch Teutschen Spraach: Grammatica. Straßburg 1574:26. Zit. in Doleschal

8. Gueintz Christian. Deutsche Sprachlehre, Entwurf. Cöthen, 1641:34-35.Zit. in Doleschal

9. Grimm Jacob. Deutsche Grammatik. Gütersloh 1837/1898.Zit.in Doleschal

10. Deutsche Forschungsgemeinschaft und UniversitätdesSaarlandeshttps://www.dfg.de/download/ pdf/foerderung/grundlagen_dfg_foerderung/ chancengleichheit/zwischenberichte/ gleichstellungsstandards_saarland_uni_2011.pdf. Zugegriffen: 16. Sept. 2021.

11. Bayerisches Staatsministerium des Innern, für Sport und Integration (2021) Freundlich, korrekt und klar - Bürgernahe Sprache in der Verwaltung, 3. Aufl.

\section{Wie sich Corona auf sexuell übertragbare Infektionen auswirkt}

Im letzten Jahr ist die Zahl der gemeldeten Infektionen mit Hepatitis $\mathrm{C}$ und HIV deutlich gesunken. Das Robert-Koch-Institut äußert beim Hauptstadtkongress 2021 die Sorge, dass sich die Zahl der unbewusst infizierten Personen vermehrt.

Insgesamt wurden während der globalen Corona-Pandemie 20\% weniger HIVErstdiagnosen im Vergleich zum Vorjahr gemeldet. Gleichzeitig ist auch die Nachfrage nach dem Medikament, mit dem sich eine HIV-Infektion vorbeugen lässt, Präexpositionsprophylaxe (PrEP), gesunken. Ähnliche Entwicklungen lassen sich bei den gemeldeten Fällen von Hepatitis C beobachten, die 2020 einen klaren Rückgang aufzeigten. Das RKI geht davon aus, dass die Situation in diesem Fall vermutlich vor allem auf die Unterdiagnose und Untererfassung von Gruppen wie Drogenkonsumenten, Häftlingen und Migranten zurückzuführen sei.

Die HIV-Erstdiagnosen könnten einerseits aufgrund der reduzierten Treffen mit Sexualpartnern und einer insgesamt geringeren Mobilität zurückgegangen sein, doch man müsse bedenken, dass gleichzeitig auch Angebot und Nachfrage von HIV-Tests gesunken seien. Dies könnte dazu führen, dass in Deutschland die Anzahl von HIV-Infizierten steigt, die nichts von ihrer Infektion wissen.

In diesem Zusammenhang wurde auf dem Hauptstadtkongress daran erinnert, dass Deutschland die Internationalen Ziele der UNAIDS im Jahr 2020 verfehlt hatte. Denn statt den angestrebten mindestens $90 \%$ haben nur rund $88 \%$ der HIV-Infizierten eine Diagnose, was bedeutet, dass eine HIV-Infektion immer noch zu oft übersehen wird. Das steigert schlussendlich die Gefahr, dass die Gruppe der unbewusst HIV-positiven Menschen wächst und diese unbewusst weitere Personen infizieren.

Quelle: Ärzte Zeitung
(www.aerztezeitung.de) 